\title{
A Multi-Model Approach to Design a Robust SVC Damping Controller Using Convex Optimization Technique to Enhance the Damping of Inter-Area Oscillations Considering Time Delay
}

\author{
Abdlmnam Abdlrahem¹, Hani Albalawi² \\ ${ }^{1}$ Holcombe Department of Electrical and Computer Engineering, Clemson University, Clemson, USA \\ ${ }^{2}$ Department of Electrical and Computer Engineering, University of Tabuk, Tabuk, Saudi Arabia \\ Email: aabdlra@g.clemson.edu, halbala@ut.edu.sa
}

How to cite this paper: Abdlrahem, A. and Albalawi, H. (2017) A Multi-Model Approach to Design a Robust SVC Damping Controller Using Convex Optimization Technique to Enhance the Damping of Inter-Area Oscillations Considering Time Delay. Energy and Power Engineering, 9, 750-771.

https://doi.org/10.4236/epe.2017.912047

Received: October 13, 2017

Accepted: November 17, 2017

Published: November 20, 2017

Copyright () 2017 by authors and Scientific Research Publishing Inc. This work is licensed under the Creative Commons Attribution International License (CC BY 4.0).

http://creativecommons.org/licenses/by/4.0/

\begin{abstract}
This paper introduces a multi-model approach to design a robust supplementary damping controller. The designed fixed-order supplementary damping controller adjusts the voltage reference set point of SVC. There are two main objectives of the controller design, damping low frequencies oscillations and enhancing power system stability. This method relies on shaping the closed-loop sensitivity functions in the Nyquist plot under the constraints of these functions. These constraints can be linearized by choosing a desired open-loop transfer function. The robust controller is designed to minimize the error between the open-loop of the original plant model and the desired transfer functions. These outcomes can be achieved by using convex optimization methods. Convexity of the problem formulation ensures global optimality. One of the advantages of the proposed approach is that the approach accounts for multi-model uncertainty. In contrast to the methods available in the literature, the proposed approach deals with full-order model (i.e., model reduction is not required) with lower controller order. The issue of time delay of feedback signals has been addressed in this paper for different values of time delay by applying a multi-model optimization technique. The proposed approach is compared to other existing techniques to design a robust controller which is based on $\mathrm{H}_{2}$ under pole placement. Both techniques are applied to the 68-bus system to evaluate and validate the robust controller performance under different load scenarios and different wind generations.
\end{abstract}

\section{Keywords}

$\mathrm{H}_{\infty}$, Nyquist Diagram, Inter-Area Modes, Multi-Model, Oscillations, Robust 


\section{Introduction}

The power system grid has increased rapidly, something which has added additional challenges to the process of reliable power transfer between interconnected systems in a large power network. The large-scale penetration of intermittent renewable energy increases uncertainty and variability to power system operation. For secure operation of power systems under variable conditions, a power system's damping controllers must be robust. Electromechanical oscillations in the range of $0.2 \mathrm{~Hz}$ to $1 \mathrm{~Hz}$ are categorized as inter-area modes. These modes primarily arise due to weak interconnections characterized by long transmission lines between different operating areas of an interconnected power system. One of the main challenges in the secure operation of an interconnected power system is the damping of these inter-area modes [1]. System stability could be affected without adequate damping of these low frequency oscillations. Event such as the 1996 western interconnect blackout is an example of one such event.

Recently, Flexible AC Transmission System (FACTS) devices have become widely used in power systems. The main purpose of using these devices is to increase the capability of transferred power between interconnected areas and enhance the voltage profile. A Static Var Compensator (SVC) is a shunt FACTS device which injects reactive power to maintain the voltage at a point of connection within a particular range to enhance system stability. Controlling SVCs helps to damp inter-area oscillations. A supplementary signal could be added to adjust the voltage reference set point of SVC to achieve the desired damping, [2] [3]. The location of SVCs for damping inter-area oscillation is important and the SVCs are usually placed at either end of a tie-line, [4] [5]. Depending on system configuration, multiple SVCs may be needed to improve overall system damping.

Recently, researchers have investigated $\mathrm{H} 2, \mathrm{H}_{\infty}$ optimizations [6] [7] [8] and $\mu$-synthesis [9] in power systems to design a robust controller. These approaches are mainly used to improve power system stability and damp power system oscillations. Riccati's equation is used in these approaches to solve the $\mathrm{H}_{\infty}$ control design problem. Recently, the linear matrix inequalities (LMIs) approach has been used to solve this issue and has provided better performance. The new techniques are presented in [10] [11] and show how to design a robust controller for multi-model uncertainty using $\mathrm{H}_{2}$ and $\mathrm{H}_{\infty}$ under pole placement; however, these techniques require reducing the order of the plant model.

The challenges of the existing approaches are as follows:

1) These approaches are based on reducing system order. The model reduction is the process of reducing the order of a given plant model in which the 
performance of the reduced model is identical to the original one [12] [13]. As a result, there is a loss of information. The level of this information loss depends on the order to which the system is reduced and the method used to reduce the information. The proposed method does not require any model order reduction. In addition, model order reduction is an $\mathrm{O}\left(\mathrm{n}^{\wedge} 3\right)$ operation and the computing model order reduction for large systems is computationally expensive.

2) The order of the controller based on existing approaches is usually high for large systems [4]-[10] since it is the sum of the orders of the reduced plant model plus the order of the weights filter as mentioned in [14]. For example, in reference [15] the order of the controller is 10 and it is 7 in reference [10]. Despite using an unreduced plant model, the proposed controller results in a lower order controller than existing methods. For the case study, the controller designed using the $\mathrm{H}_{2}$ under pole placement technique resulted in a 9th order controller. Using the proposed approach resulted in a 4th order controller with similar or better performance.

3) In most existing methods, the controller is designed based on one operating point [4]-[16], i.e., the robustness is guaranteed around this operating point, but is not guaranteed if the system operates far from this point. A power system is a non-stationary system where operating points change for every dispatch at the system operator level. Performance of the controller degrees depend on the deviation between the current operating point and the nominal operating point for which the controller was designed.

Considering these challenges, the contributions of this paper are outlined below:

- The entire plant model is used and there is no need for model order reduction.

- The resulting controller order is less than the controller order used in other existing methods.

- Multi-model uncertainty is considered.

- Time delay of remote signals is accounted for.

- Convex formulation guarantees global optimal solutions while minimizing the error between open-loop and desired transfer functions.

This paper is organized as follows:

- Section 2 discusses controller design.

- Section 3 describes the test system with SVC and DFIG.

- Section 4 describes the step-by-step controller design procedures.

- Section 5 shows and discusses simulation results.

- Section 6 presents conclusions.

\section{2. $H_{\infty}$ Robust Controller Design}

\subsection{Class of Controller}

A linear parameterized controller can be represented as:

$$
K(s)=\rho^{\mathrm{T}} \varphi(s)
$$


where, $\rho=\left[\rho_{1} \rho_{2} \cdots \rho_{n}\right]$

$$
\varphi(s)=\left[\varphi_{0}(s) \varphi_{1}(s) \cdots \varphi_{n-1}(s)\right]^{\mathrm{T}}
$$

and $\rho_{i}$ is the controller parameters, $n$ is the number of controller parameters and $\varphi_{i}(s)$ is a basis function. One of the common basis functions is the Laguerre basis function given in (2) [17].

$$
\varphi_{0}(s)=1, \varphi_{i}(s)=\frac{\sqrt{2 \zeta}(s-\zeta)^{i-1}}{(s+\zeta)^{i}}, i \geq 1, \zeta>0
$$

where $\zeta>0$. It can be shown that for any finite order transfer function $F(s)$, arbitrary Laguerre parameter $\zeta>0$ and an arbitrary constant $\varepsilon>0$ there is a finite $n$ such that

$$
\left\|F(s)-\rho^{\mathrm{T}} \varphi(s)\right\|_{p}<\varepsilon \text { for } 0<p<\text { infinity }
$$

Any finite order stable transfer function can be approximated using controller parameterization in (1) with a desired level of accuracy by changing the number of controller parameters $n$. A good approximation of $F(s)$ can be achieved for a given controller order if the choice of $\zeta$ is proper. For more details of choosing the basic functions see [17].

To obtain a convex parameterization of a fixed order controller, the linearly parameterized controller in (1) is used. The reason for the use of (1) is that all the points of the open-loop transfer function $L(j \omega, \rho)$ on the Nyquist plot can be written as a linear function of the controller parameters $\rho$ as given in (4).

$$
L(j \omega, \rho)=K(j \omega, \rho) G(j \omega)=\rho^{\mathrm{T}} \varphi(j \omega) G(j \omega)=\rho^{\mathrm{T}} \mathcal{R}(\omega)+j \rho^{\mathrm{T}} \mathcal{J}(\omega)
$$

where $\mathcal{R}(\omega)$ and $\mathcal{J}(\omega)$ are the real and imaginary parts of $\varphi(j \omega) G(j \omega)$.

The plant model $G$ is a scalar function used in the case of a single model and in the case of a multi-model controller, a set of the multi-model uncertainty can be defined as $\mathcal{G}=\left\{G_{i}(j \omega), i=1, \cdots, m\right\}$ where $G_{i}(j \omega)$ represents the i-th model in this set. $L_{i}(j \omega)$ represents the open-loop transfer function for the i-th model.

\subsection{Uncertainty and Robustness Representation}

\subsubsection{Multiplicative Uncertainty}

Multiplicative uncertainty is represented in (5). Suppose that $G_{0}(j \omega)$ is the normal plant frequency response and the actual plant that describes the normal plant with uncertainty is $G(j \omega)$ as shown in Figure 1 and (5) [18].

$$
G(s)=G_{0}(s)\left(1+W_{2}(s) \Delta(s)\right)
$$

where $\Delta(s)$ is an unknown stable transfer function with $\|\Delta\|_{\infty}<1$.

\subsubsection{Robust Stability and Performance.}

The closed loop system in Figure 1 can be represented by:

$$
y=\frac{K(s) G(s)}{1+K(s) G(s)}(r-n)+\frac{1}{1+K(s) G(s)} d
$$




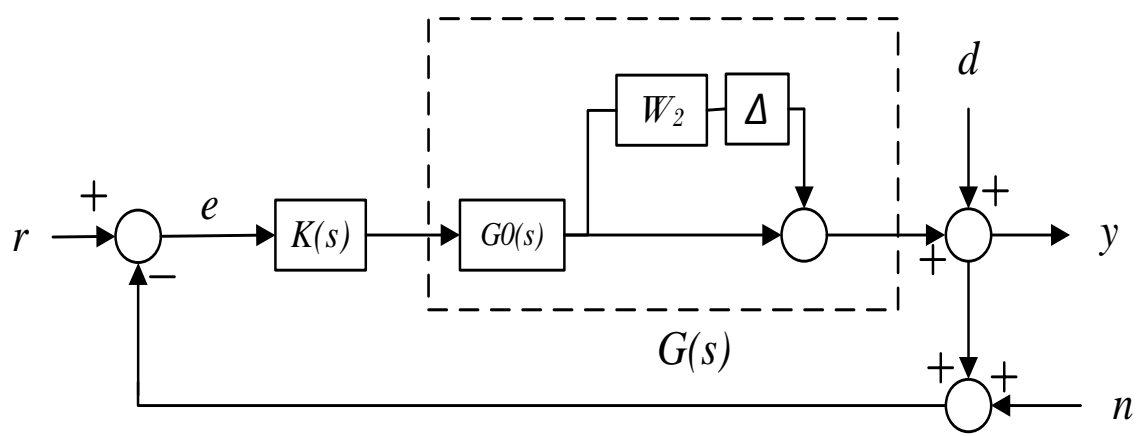

Figure 1. Black diagram representing an uncertain feedback system.

Let the open loop transfer function $L(j \omega)=K(j \omega) G(j \omega)$, the complementary sensitivity function $T(j \omega)=L(j \omega) /[1+L(j \omega)]$, and the sensitivity function $S(j \omega)=1 /[1+L(j \omega)]$ be defined. It can be seen from (6) that $T(j \omega)$ defines the relationship between the reference and the output signals. $S(j \omega)$ defines the relationship between the reference and the error. These transfer functions define the main characteristic of the closed loop architecture.

The Nyquist diagram has been used to derive the criteria of robust performance as well as the robust stability. The point $(-1+j 0)$ on the Nyquist plot shown in Figure 2 is known as the critical point that is used to study the closed-loop system stability. The circle centered at the critical point $(-1+j 0)$ with radius $W_{1}(j \omega)$ is known as the performance disc. The uncertainty disc is represented by the circle with radius $W_{2}(j \omega) L(j \omega, \rho)$.

Graphically, robust stability is achieved if, and only if, the uncertainty disc centered at the original open-loop transfer function with radius $W_{2}(j \omega) L(j \omega, \rho)$ does not intersect with the other circle centered at the critical point $(-1+j 0)$ with radius $W_{1}(j \omega)$ on the Nyquist plot. $|1+L(j \omega, \rho)|$ defines the distance between the center of the critical point and the center of the uncertainty disc. For robust stability, the radius $W_{2}(j \omega) L(j \omega, \rho)$ of the uncertainty circle has to be less than the distance $|1+L(j \omega, \rho)|$ at all frequencies. In other words, $\left|W_{2}(j \omega) L(j \omega)\right|<|1+L(j \omega, \rho)|$ is used for all $\omega$. Dividing both sides of this equation by $|1+L(j \omega, \rho)|$ and by knowing the fact $T(j \omega)=L(j \omega) /[1+L(j \omega)]$ the following is obtained:

$$
\left|W_{2}(j \omega) T(j \omega)\right|<1 \quad \forall \omega
$$

The normal performance condition of stable system can be given in the following standard form [18].

$$
\left|W_{1}(j \omega) S(j \omega)\right|<1 \quad \forall \omega
$$

To define the condition of the robust performance of the system given in Figure 2 , substitute (5) in to (8), as given in (9):

$$
\left|W_{1} S\right|=\left|\frac{W_{1}}{1+(1+\Delta) L}\right|=\left|\frac{W_{1} S}{1+\Delta T}\right|<\left|\frac{W_{1} S}{1-W_{2} T}\right|
$$




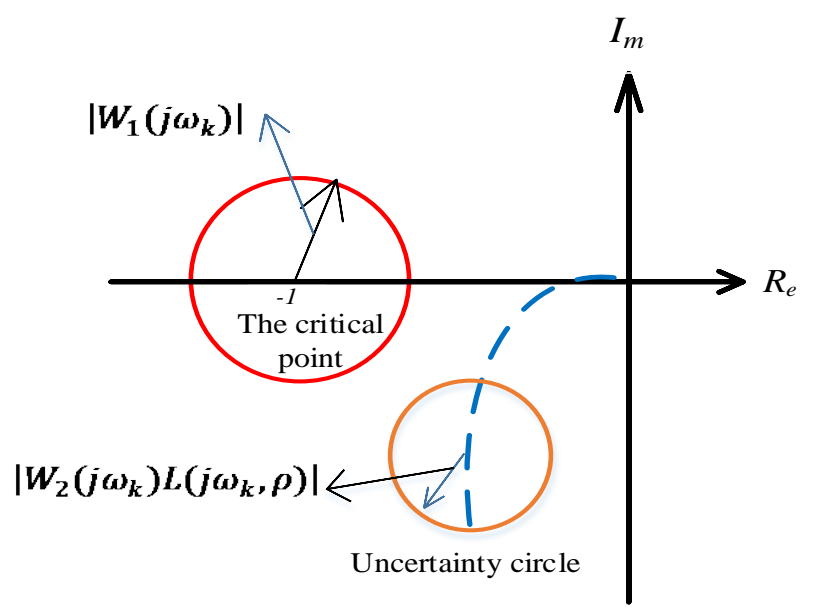

Figure 2. Nyquist plot.

Since $\left|W_{1} S\right|<1$, then $\left|\frac{W_{1} S}{1-W_{2} T}\right|<1$ from the above equation. This constraint is required for robust performance, and, by rearranging this constraint, the standard form of the robust performance is obtained as provided in (10):

$$
\left|W_{1}(j \omega) S(j \omega)\right|+\left|W_{2}(j \omega) T(j \omega)\right|<1 \quad \forall \omega
$$

\subsubsection{The Proposed Approach}

The constraints in (10) satisfy the robust stability as well as robust performance. These constraints are represented in the Nyquist plot. Robustness can be achieved by using a set of convex constraints on the frequency domain. The controller can be designed based on a convex optimization problem. The solution to this issue is to reduce the norm of the distance between the actual $L_{i}\left(j \omega_{k}, \rho\right)$ and desired $L_{d}\left(j \omega_{k}\right)$ open-loop transfer function as shown in Figure 3 [17].

Multiplying (10) by $|1+L(j \omega, \rho)|$ results in:

$$
\left|W_{1}(j \omega)\right|+\left|W_{2}(j \omega) L(j \omega, \rho)\right|<|1+L(j \omega, \rho)| \forall \omega
$$

The constraints in (11) are non-convex and $L_{d}\left(j \omega_{k}\right)$ are used to linearize these constraints. By making the problem convex, global optimality is ensured. Line $d$ is introduced as shown in Figure 3 which is tangent to the performance disc centered at $(-1+j 0)$ and orthogonal to the line links at the center of the performance disc to $L_{d}\left(j \omega_{k}\right)$. A sufficient condition for constraints in (11) is that the circle centered at the actual open-loop transfer function $L_{i}\left(j \omega_{k}, \rho\right)$ has to be on the right side of line $d$ for all frequencies as shown in Figure 3.

Line $d$ is a straight line in the complex plane and can be represented by an infinite number of points. Each point in the complex plane has a real part $x$ and imaginary part $y$. The equation of the straight line $d$ is a function of $L_{d}\left(j \omega_{k}\right)$ and $W_{1}$. The line can be written at each point as:

$$
\text { Line } d: y=\tan (\alpha)\left[x-\frac{\left|W_{1}\right|}{\sin (\alpha)}+1\right]
$$




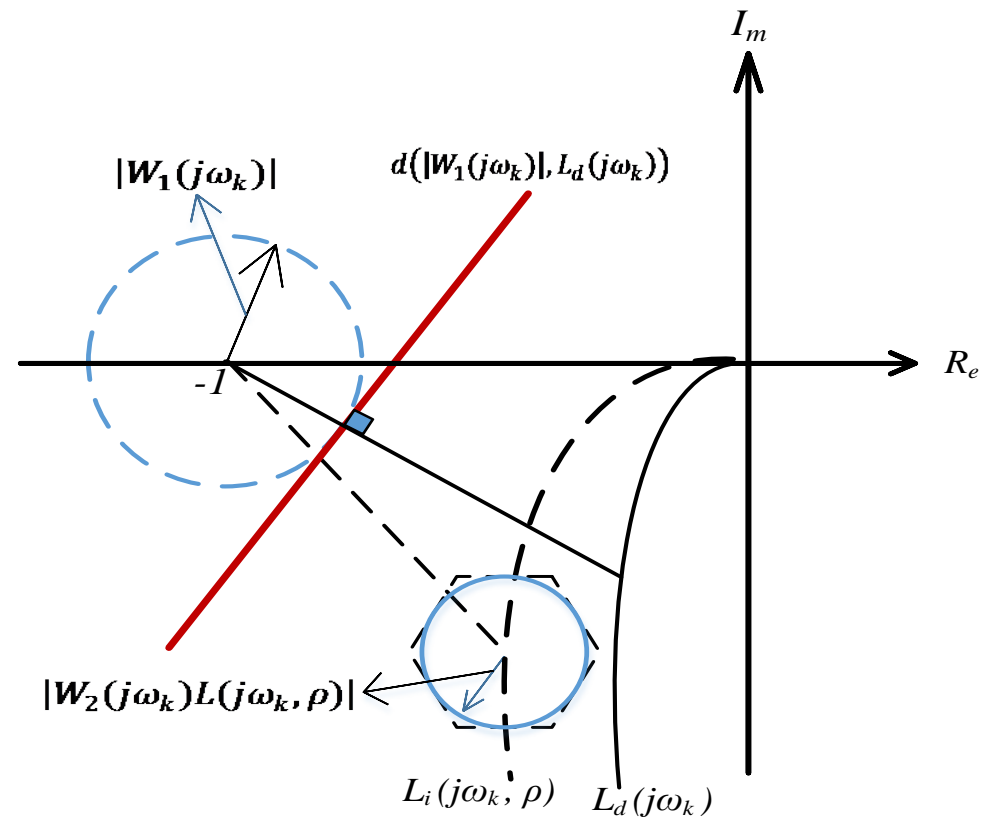

Figure 3. Linear constraints on Nyquist plot.

where $\sin (\alpha)$ and $\cos (\alpha)$ are:

$$
\sin (\alpha)=\frac{R_{e}\left\{1+L_{d}\left(j \omega_{k}\right)\right\}}{\left|1+L_{d}\left(j \omega_{k}\right)\right|}, \cos (\alpha)=-\frac{I_{m}\left\{1+L_{d}\left(j \omega_{k}\right)\right\}}{\left|1+L_{d}\left(j \omega_{k}\right)\right|}
$$

By substituting the $\sin (\alpha)$ and $\cos (\alpha)$ into the Equation (12), the following is obtained:

$$
\left|W_{1}\left(j \omega_{k}\right)\left[1+L_{d}\left(j \omega_{k}\right)\right]\right|-I_{m}\left\{L_{d}\left(j \omega_{k}\right)\right\} y-\left[1+R_{e}\left\{L_{d}\left(j \omega_{k}\right)\right\}\right][1+x]=0
$$

The linear constraints of line $d$ that exclude the performance disk are given in (14):

$$
\begin{aligned}
& \left|W_{1}\left(j \omega_{k}\right)\left[1+L_{d}\left(j \omega_{k}\right)\right]\right|-I_{m}\left\{L_{d}\left(j \omega_{k}\right)\right\} I_{m}\left\{L\left(j \omega_{k}, \rho\right)\right\} \\
& -\left[1+R_{e}\left\{L_{d}\left(j \omega_{k}\right)\right\}\right]\left[1+R_{e}\left\{L\left(j \omega_{k}, \rho\right)\right\}\right]<0 \quad \forall \omega
\end{aligned}
$$

The linear constraints in (14) can be written in a simpler way using the facts:

$$
R_{e}\left\{L_{d}\left(j \omega_{k}\right)\right\}=1 / 2\left[L_{d}\left(j \omega_{k}\right)+L_{d}^{*}\left(j \omega_{k}\right)\right]
$$

and

$$
I_{m}\left\{L_{d}\left(j \omega_{k}\right)\right\}=1 / 2\left[L_{d}\left(j \omega_{k}\right)-L_{d}^{*}\left(j \omega_{k}\right)\right]
$$

The constraints in (14) become:

$$
\left|W_{1}\left(j \omega_{k}\right)\left[1+L_{d}\left(j \omega_{k}\right)\right]\right|-R_{e}\left\{\left[1+L_{d}^{*}\left(j \omega_{k}\right)\right]\left[1+L\left(j \omega_{k}, \rho\right)\right]\right\}<0 \quad \forall \omega
$$

where $L_{d}^{*}\left(j \omega_{k}\right)$ is the complex conjugate of $L_{d}\left(j \omega_{k}\right)$.

To satisfy the condition in (15) for a set of uncertainty models, the circle centered at $L_{i}\left(j \omega_{k}, \rho\right)$ should be approximated by a polygon with $v>2$ vertices. To satisfy the robust uncertainty in (10), all the vertices of the polygon located at 
the uncertainty disk have to be on the right side of the line $d$. This condition can be represented by the linear constraints of (16):

$$
\left|W_{1}\left(j \omega_{k}\right)\left[1+L_{d}\left(j \omega_{k}\right)\right]\right|-R_{e}\left\{\left[1+L_{d}^{*}\left(j \omega_{k}\right)\right]\left[1+L_{i}\left(j \omega_{k}, \rho\right)\right]\right\}<0 \forall \omega
$$

where $L_{i}\left(j \omega_{k}, \rho\right)=K\left(j \omega_{k}, \rho\right) G_{i}(j \omega)$, and

$$
G_{i}(j \omega)=G(j \omega)\left[1+\frac{\left|W_{2}\left(j \omega_{k}\right)\right|}{\cos (\pi / v)} \mathrm{e}^{-2 j \pi i / v}\right]
$$

It is observed that the number of the linear constraints is multiplied by $v$.

There is another way to satisfy the robust condition in (11) by increasing the radius of the circle $\left|W_{2}(j \omega) L(j \omega, \rho)\right|$ which leads to the following convex constraints:

$$
\begin{aligned}
& \left|W_{1}\left(j \omega_{k}\right)\left[1+L_{d}\left(j \omega_{k}\right)\right]\right|+\left|W_{2}(j \omega) L(j \omega, \rho)\right|\left[1+L_{d}\left(j \omega_{k}\right)\right] \\
& -R_{e}\left\{\left[1+L_{d}^{*}\left(j \omega_{k}\right)\right]\left[1+L_{i}\left(j \omega_{k}, \rho\right)\right]\right\}<0 \quad \forall \omega
\end{aligned}
$$

Considering these examinations, the quadratic optimization problem can be expressed as given in (19):

$$
\min _{\rho} \sum_{i=1}^{m} \sum_{k=1}^{N_{i}}\left|L_{i}\left(j \omega_{k}, \rho\right)-L_{d}\left(j \omega_{k}\right)\right|^{2}
$$

Subject to:

$$
\begin{aligned}
& \left|W_{1}\left(j \omega_{k}\right)\left[1+L_{d}\left(j \omega_{k}\right)\right]\right|+\left|W_{2}(j \omega) L(j \omega, \rho)\right|\left[1+L_{d}\left(j \omega_{k}\right)\right] \\
& -R_{e}\left\{\left[1+L_{d}^{*}\left(j \omega_{k}\right)\right]\left[1+L_{i}\left(j \omega_{k}, \rho\right)\right]\right\}<0 \quad \forall \omega
\end{aligned}
$$

for $k=1, \cdots, N_{i}$ (No. of frequencies), $i=1, \cdots, m$.

where $L_{i}\left(j \omega_{k}, \rho\right)=\rho^{\mathrm{T}} \varphi\left(j \omega_{k}\right) G_{i}\left(j \omega_{k}\right)$

For multi-model uncertainty case, the constraints in (18) can be repeated for all the plant models $G_{i}(j \omega)$ for $i=1, \cdots, m$. The constraints in (18) still can be used if the uncertainty weights filter $W_{1}, W_{2}$ and the desired open-loop transfer function, $L_{d i}$, is different for each plant model since these constraints are convex with respect to $G_{i}(j \omega)$ for multi-model uncertainty.

\section{The IEEE 68-Bus Test System and SVC Model}

\subsection{Test System}

The IEEE16 machines, 68-bus system is used in this paper. This test system is particularly suited for small signal stability studies. For instance, reference [14] uses the same test system for damping inter-area modes. There are five distinct areas in the test system with a total load of $18.23 \mathrm{GW}$. Areas NETS and NYPS are interconnected through two parallel tie-lines. Figure 4 shows the single line diagram of the test system. Parameters of the generators, exciters, governors, and transmission lines of the test system can be found in [14].

Power System Toolbox (PST) [19] is used to simulate the test system including the SVC and doubly-fed induction generator (DFIG). The controller was implemented in MATLAB based on the proposed approach and has been integrated in PST. 


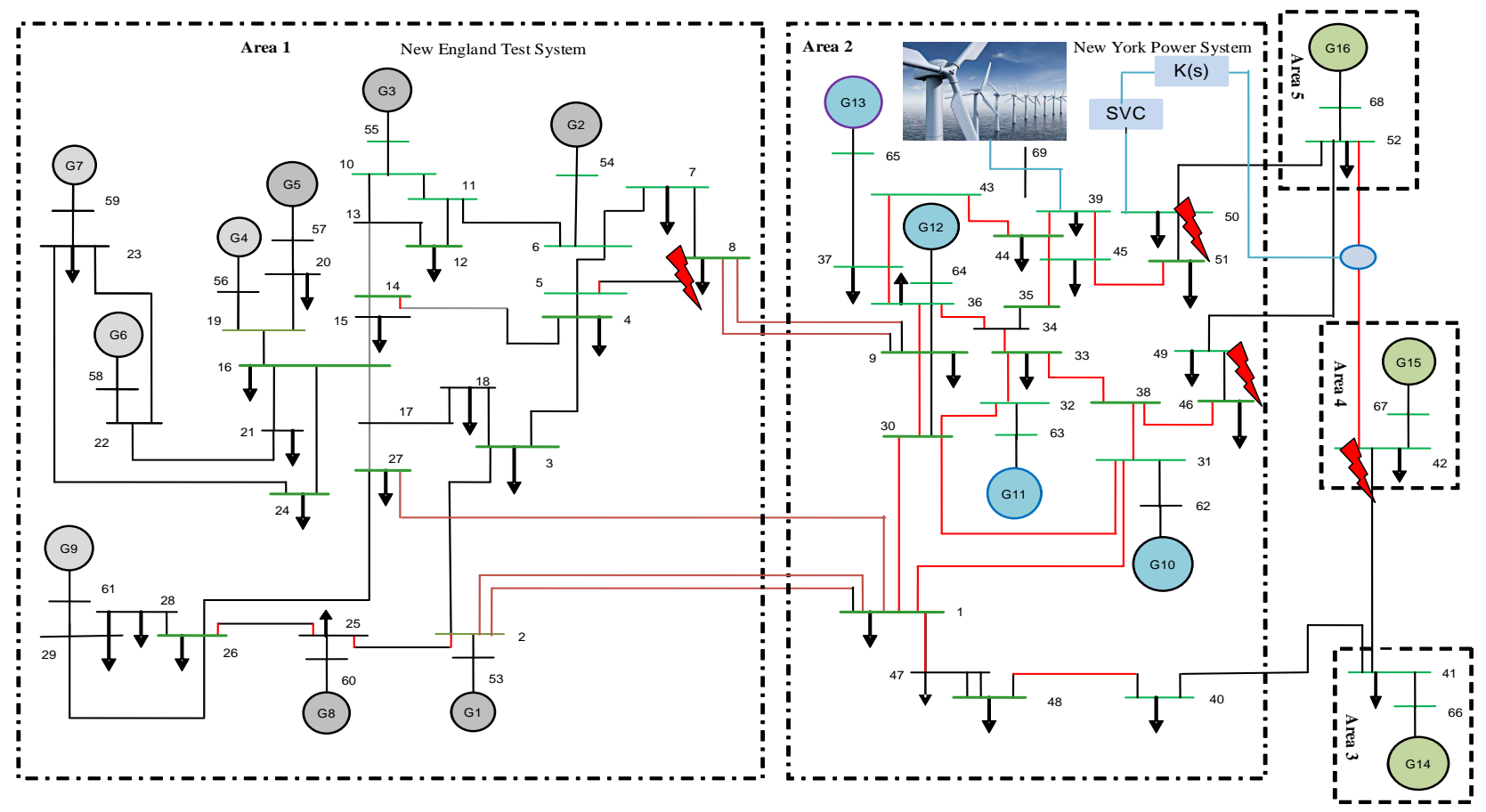

Figure 4. Single line diagram of 68 bus test system.

To include renewable generation, a $500 \mathrm{MW}$ wind farm is placed in area 2 at bus 39 as presented in Figure 4. The wind farm is installed to add more variability to the system due to the continuous change of the output power of the wind farm. The third order model of a DFIG is used. The dynamic model of the DFIG contains a set of differential algebraic equations that has been integrated in PST [20]. A single model of DFIG is used to represent the wind farm.

\subsection{Static Var Compensator}

The block diagram of SVC is shown in Figure 5(a). The test system has an SVC installed at bus 50. Parameters of SVC are given in Table 1. The objective of designing the controller is to damp tie line oscillations by providing additional signal to the set point of the SVC. Control structure of the proposed approach is represented as shown in Figure 5(b).

\section{Controller Design Procedure}

In this section, the step by step procedure and rationale used in designing the controller is described in detail.

\subsection{Selecting Inter-Area Modes}

For the given test system, under nominal operating condition, two eigenvalue pairs have damping less than $5 \%$. In fact, one of the eigenvalue pairs has damping very close to zero, hence, the system is close to instability point. Based on the eigenvalues for nominal operating point, the inter-area modes that need to be damped for the case study are listed in Table 2. Figure 6 shows the damping ratios for frequencies of interest corresponding to the nominal operating condition. 


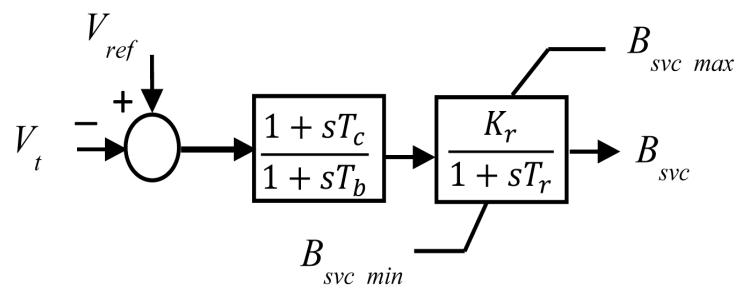

(a)

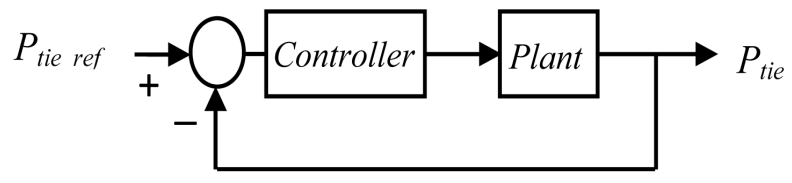

(b)

Figure 5. The block diagram of (a) SVC and (b) control representation.

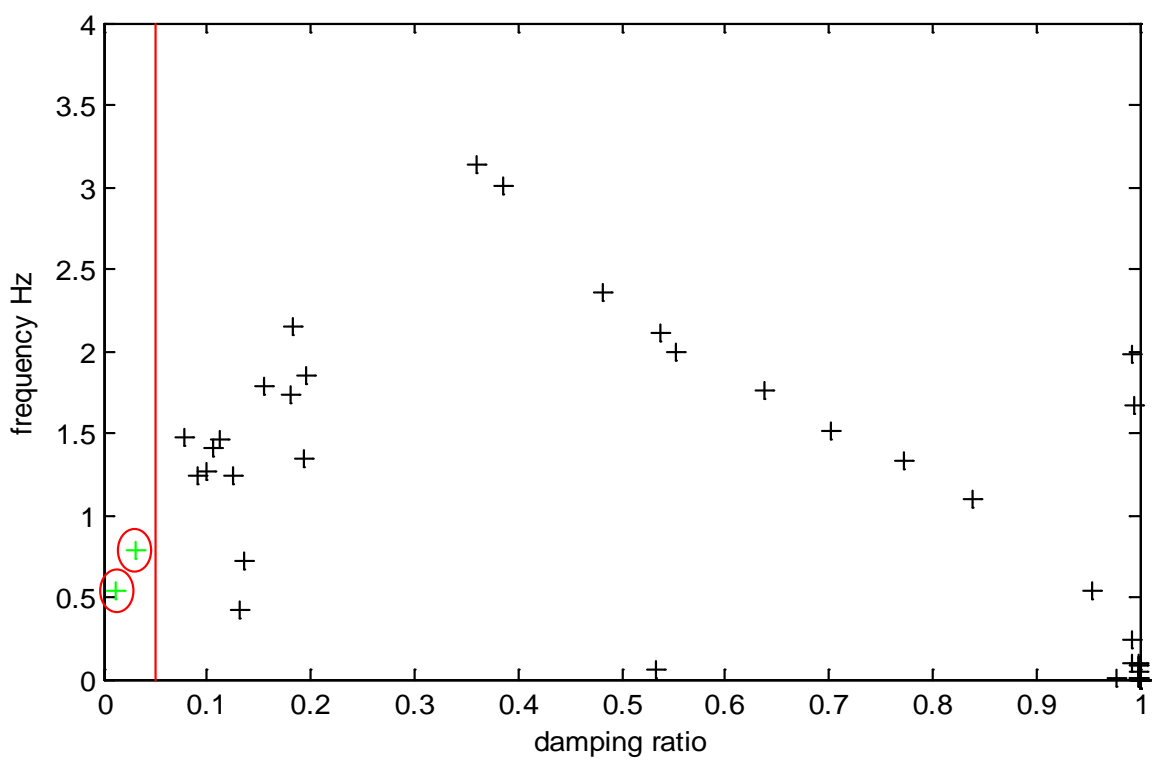

Figure 6. Damping ratios and frequencies of eigenvalues for OP1, normal operating point.

Table 1. SVC parameters.

\begin{tabular}{cccccc}
\hline$B_{\text {svc } \max }$ & $B_{\text {svc min }}$ & $K_{r}$ & $T_{r}$ & $T_{c}$ & $T_{b}$ \\
\hline $1 \mathrm{pu}$ & $1 \mathrm{pu}$ & 10 & $0.05 \mathrm{sec}$ & $0.6 \mathrm{sec}$ & $0.2 \mathrm{sec}$ \\
\hline
\end{tabular}

Table 2. Eigenvalues, damping ratios, and the frequencies of the inter-area modes of the test system.

\begin{tabular}{ccc}
\hline $\begin{array}{c}\text { Eigenvalue } \\
\sigma \pm j \omega\end{array}$ & Damping ratio & Frequency $(\mathrm{Hz})$ \\
& $-\frac{\sigma}{\sqrt{\sigma^{2}+\omega^{2}}}$ & $\frac{\omega}{2 \pi}$ \\
\hline$-0.04052 \pm 3.410 j$ & 0.01188 & 0.5427 \\
$-0.1539 \pm 4.948 j$ & 0.03108 & 0.7875 \\
\hline
\end{tabular}




\subsection{Selecting Input/Output Signal}

Appropriate selection of the input signal for the designed controller is highly essential to guarantee that the inter-area eigenvalues are controllable and observable. The controllability metric is used to choose the best effective input signal to damp the inter-area modes. The controllability metric is defined as the amount of displacement that a pole would undergo due to small change in the feedback gain and this is given in (20) [21]. Using the controllability metric as shown in Figure 7, the active power flow of the line 42 to 52 is found as the most controllable measurement to damp the inter-area modes. Therefore, the input signal that feeds the controller is provided from the tie-line (42 to 52), which connects areas 4 and 5 . The controller output is used as additional control signal to the SVC.

$$
\Delta \lambda_{i}=u_{i} B \Delta K C v_{i} \rightarrow \frac{\left\|\Delta \lambda_{i}\right\|}{\|\Delta K\|} \leq\left\|u_{i} B\right\| *\left\|C v_{i}\right\|
$$

\subsection{Choice of Operating Points}

A power system is a non-stationary system where a set of new dispatches are computed every 5 to 15 minutes. As a result, the total number of possible operating points are innumerable, hence, six different operating points which represent several stress levels of the system are used for controller design and validation. Stress levels of the system in this context are quantified using eigen-spectrum. Eigenvalues convey two very important attributes: oscillation frequencies and their corresponding damping ratio. Damping ratio illustrates how much energy is dissipated during each cycle for a given frequency.

Six different operating points are created where the damping ratio of the eigenvalues that correspond to inter-area mode of the system are progressively made worse. The system has been extensively studied and these operating points listed in Table 3 are considered for this study as they greatly affect the inter-area modes. The generators G15 and G16 are adjusted to obtain different operating points. In addition, wind generation also varies between different operating points. All the values in Table 3 are in per-unit system.

\subsection{Desired Open Loop Transfer Function $\left(L_{d}\right)$}

Selecting $L_{d}$ is based on design specifications. $L_{d}$ normally has a high amplitude in low frequencies for good tracking and the system follows the reference signal. At high frequencies, $L_{d}$ should have a small amplitude to provide robustness and noise rejection characteristics. $L_{d}(s)$ could be chosen as $\omega_{c} / s$ where $\omega_{c}$ is the desired closed-loop bandwidth [17] [22]. Typically, the bandwidth is the range of frequencies for which the gain is significant. Generally, a high bandwidth will allow for a faster response. In the case study, the aim is to damp the inter-area modes in the range of frequencies $(0.2-1.0 \mathrm{~Hz})$, so a bandwidth of more than $2 \pi f=2 \pi * 1.0=6.28 \mathrm{rad} / \mathrm{sec}$ is needed. The desired bandwidth $\omega_{c}$ should be more than $6.28 \mathrm{rad} / \mathrm{sec}$. For the case study, there is a 


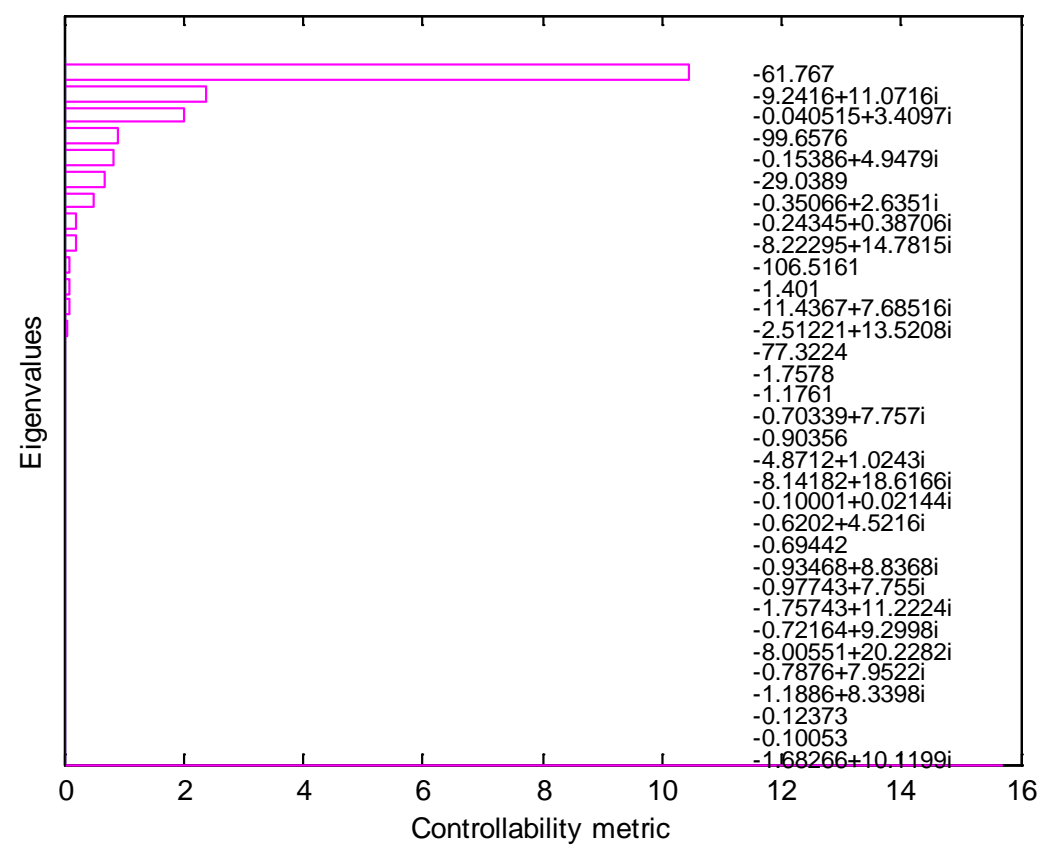

Figure 7. Controllability indices of controllable eigenvalues based on selecting the line 42 to 52 .

Table 3. Different operating points for 68 bus system.

\begin{tabular}{cccc}
\hline OP \# & Gen 15 & Gen 16 & Wind generation \\
\hline 1 & 5 & 40 & 5 (Normal model) \\
2 & 7 & 38 & 5 \\
3 & 5 & 43 & 2 \\
$4^{*}$ & 5 & 44 & 3 \\
$5^{*}$ & 5 & 42 & 3 \\
$6^{*}$ & 7 & 40 & \\
\hline
\end{tabular}

${ }^{*}$ It is not used in the control design, it is used to validate the controller.

strong resonance mode around $\omega_{1}$ and $\omega_{2}$ as shown in Figure 8 and these modes should be cancelled by the controller. So $\omega_{c}$ is selected as $\omega_{c}=9$ which means $\left(L_{d 0}=9 / s\right)$.

\subsection{Weighting Filters ( $W_{1}$ and $\left.W_{2}\right)$}

Selection of $W_{1}$ and $W_{2}$ is essential for the controller design. In this work, $W_{1}$ is designed as a first-order low-pass filter to gain a good disturbance rejection. $W_{2}$ is designed as a high-pass filter to guarantee robustness and minimize the controller effort in high frequencies. Frequency response of $W_{1}$ and $W_{2}$ is shown in Figure 9.

$$
W_{1}(s)=\frac{20}{s+10}, \quad W_{2}(s)=\frac{20 s}{s+100}
$$




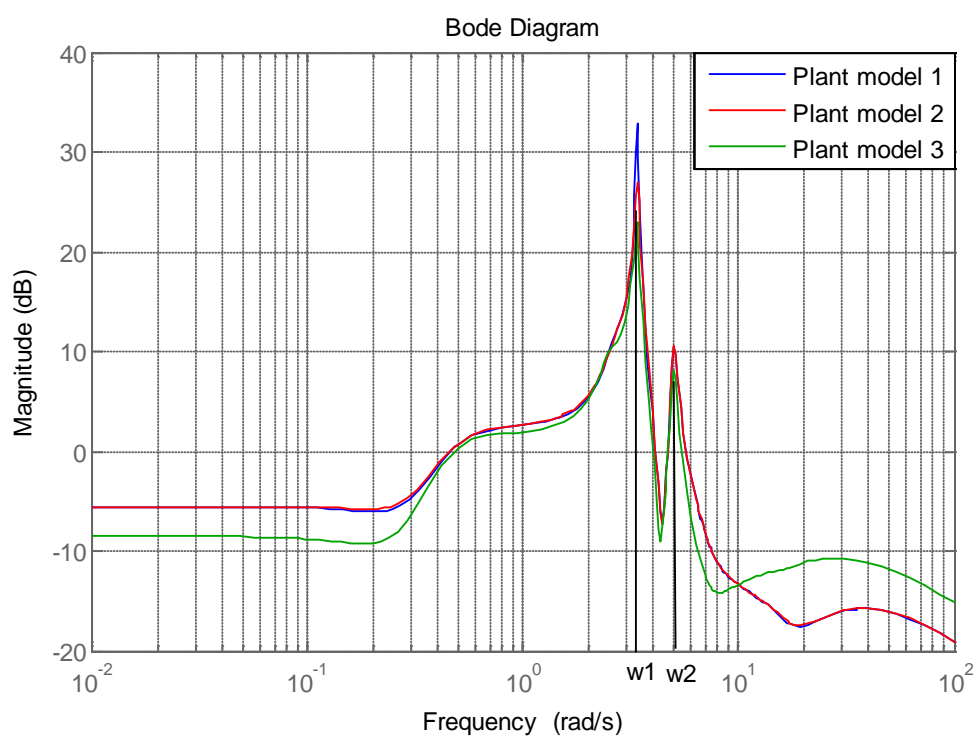

Figure 8. Frequency response of the three selected plant models.

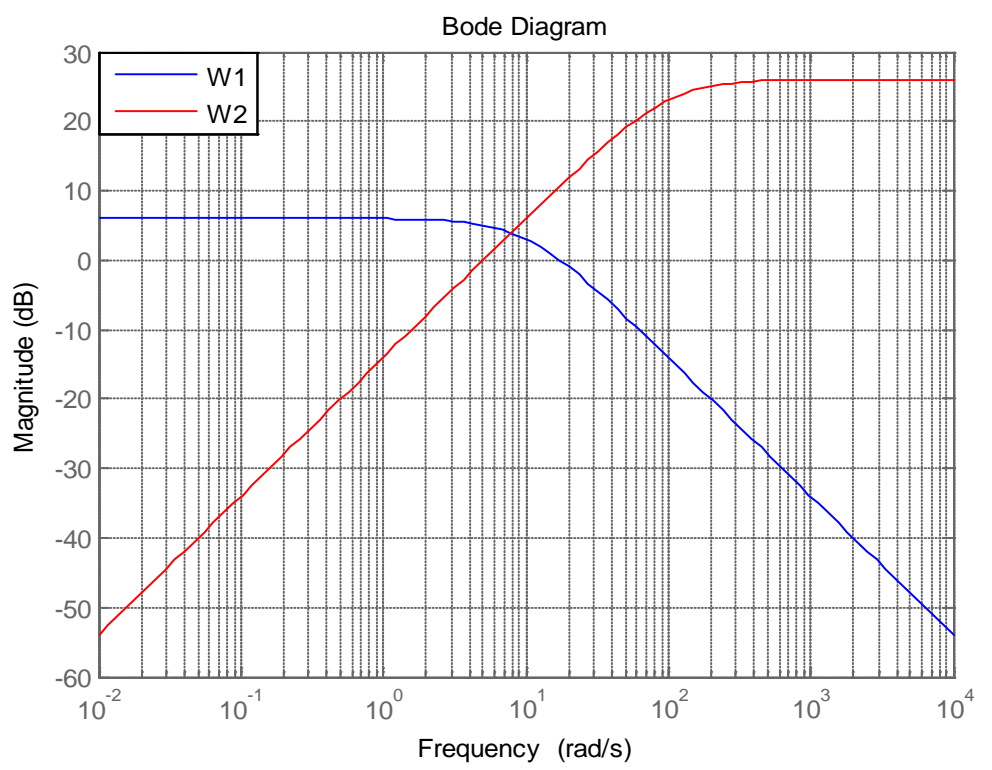

Figure 9. The frequency response of the weighting filters.

\subsection{Solving the Optimization Problem}

The convex quadratic programming problem described by (19) is solved to obtain $K_{0}(s)$. Since, the problem is convex, global optimality is guaranteed. Using the relation, $L_{d i}=K_{0} G_{i}, i=1,2,3$, the desired open-loop transfer function is computed for operating points OP1 to OP3. The three computed $L_{d}$ values with the three models are used to design the final controller $K(s)$ by solving optimization problem in (19). The final controller $K(s)$ as given in (22).

$$
K(s)=\frac{-43.095(s+17.13)(s+0.07859)\left(s^{2}+0.7662 s+6.429\right)}{(s+9)^{4}}
$$




\section{7. $\mathrm{H}_{2}$ Controller under Pole Placement}

For comparison, a damping controller is designed using pole placement and $\mathrm{H}_{2}$ optimization following two steps based on matrices [11]. First, a state feedback controller is developed that uses the system states to generate a control signal. This is achieved by solving a set of Linear Matrix Inequalities (LMIs) that places the system poles into a cone area in the complex plane, while minimizing the amplitude of control signal represented by its $\mathrm{H}_{2}$ norm. Thereafter, a state estimator is developed that constructs system states from the output. A similar set of LMIs is employed for this purpose. The controller can be obtained by a transfer function equivalence of the state-feedback controller and the state estimator combined. This approach is considered multi-model so the controller is designed based on different load conditions. However, this approach still suffers from drawbacks 1 and 2 listed in section 1 . For the case study, the damping ratio is set to be $10 \%$ as the boundary of the pole placement region. Also, the weights filter is selected to be the same as the weight filter used in the proposed approach. The same operating points listed in Table 3 are used to design the controller using this approach. Plant/system model needs to be reduced based on this approach in such a way that the response of the reduced system is similar to that of the original system in the frequency range of interest. The test system consists of 190 states including the DFIG and the SVC. For the frequency range of interest, the plant model can be reduced to at least 7th order. In addition, the total order of the controller based on [14] is equal to order of the reduced system plus the order of weighting filters. In this case, this equates to a controller order of $7+2$ i.e., 9 states. Figure 10 shows the original and the reduced plant model and the figure shows that these models are identical in the frequency range of interest. No model order reduction is required for the proposed method. Using the proposed approach, a 4 th order controller is designed which replicates the

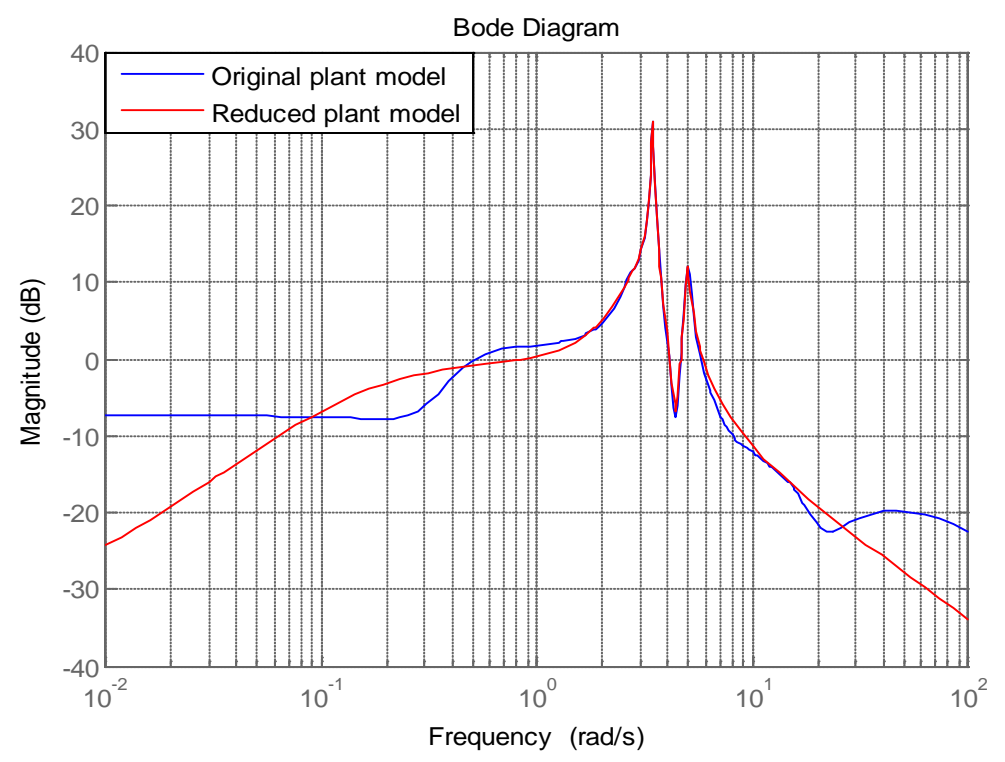

Figure 10. Frequency response of the original and the reduced system, OP1. 
frequencies of interest. This approach is applied to design a robust controller to compare it with the proposed method. Interested readers are referred to [11] for more details.

\section{Results and Discussion}

In this section, a two-part validation of the proposed approach is presented. Comparisons are drawn for the proposed method for the numerical and time-based domains, with the base case only accounts for SVC and the methods presented in section 4 . In the first part of validation, eigenvalue spectrum obtained using the different methods are compared. Specifically, comparisons for damping ratios are drawn for different modes of interest.

In the second part, time domain performance results are presented. Comparing controllers which have user-defined parameters is not straight forward. A given control methodology and design can result in a poorly performing controller based on bad parameter selection. One way to avoid this situation is to use a standard set of values for user defined parameters and use the same parameters in both approaches. The same parameters used in [11] [23], and [24] are used for $\mathrm{H}_{2}$ under pole placement controller such as the damping ratio of the boundary of the pole placement region. The same controller operating points and weights are used in both approaches. Furthermore, the focus of the validation process is not to provide a quantitative comparison, but rather to show that the proposed controller gives comparable results to those of existing methods with the advantages listed in section 1.

\subsection{Eigenvalue Analysis}

Eigenvalue comparisons of the proposed controller with base case (i.e., with only the SVC for six different operating points) are given in Table 4. Substantial improvements in damping ratio are seen with the proposed controller. For instance, when mode 1 with operating point 4 is considered, the system has no controller and the damping ratio is negative. These circumstances lead to an unstable system.

With the addition of the proposed controller, the damping ratio is improved to $(0.1814)$ from $(-0.0008)$. A similar trend of improved damping ratio is seen across all six operating points. The modes of the test system under different load conditions are shown in Figure 11.

\subsection{Time Domain Analysis}

\subsubsection{Robustness under Variability in Load Conditions and Wind Generation}

To investigate the robustness of the proposed controller, a three-phase fault is placed at different areas with different operating points. Application of a fault in power systems results in differences between mechanical and electrical power which results in electromechanical oscillations. The tests used for validating 
Table 4. Damping and frequencies of the inter-area modes under different load conditions of the 68-bus system.

\begin{tabular}{|c|c|c|c|c|c|c|c|c|}
\hline \multirow{4}{*}{$\begin{array}{l}\begin{array}{c}\text { Operating point } \\
\text { No. }\end{array} \\
1\end{array}$} & \multicolumn{4}{|c|}{ SVC } & \multicolumn{4}{|c|}{ SVC with $\mathrm{H}_{\infty}$ controller } \\
\hline & \multicolumn{2}{|c|}{ Mode 1} & \multicolumn{2}{|c|}{ Mode 2} & \multirow{2}{*}{\multicolumn{2}{|c|}{$\begin{array}{c}\text { Mode } 1 \\
\xi \xi\end{array}$}} & \multicolumn{2}{|c|}{ Mode 2} \\
\hline & \multirow{2}{*}{$\begin{array}{c}\xi \\
0.01188\end{array}$} & \multirow{2}{*}{$\begin{array}{l}\mathrm{f}(\mathrm{Hz}) \\
0.5427\end{array}$} & \multirow{2}{*}{$\begin{array}{c}\xi \\
0.03108\end{array}$} & \multirow{2}{*}{$\begin{array}{l}\mathbf{f}(\mathrm{Hz}) \\
0.7875\end{array}$} & & & $\xi$ & $\mathrm{f}(\mathrm{Hz})$ \\
\hline & & & & & 0.1935 & 0.5085 & 0.1337 & 0.7200 \\
\hline 2 & 0.01659 & 0.5448 & 0.03286 & 0.7903 & 0.1980 & 0.5103 & 0.1337 & 0.7199 \\
\hline 3 & 0.00267 & 0.5266 & 0.03026 & 0.7850 & 0.1880 & 0.4883 & 0.1335 & 0.7159 \\
\hline 4 & -0.0008 & 0.5194 & 0.03005 & 0.7838 & 0.1814 & 0.4818 & 0.1326 & 0.7141 \\
\hline 5 & 0.00596 & 0.5327 & 0.03050 & 0.7860 & 0.1921 & 0.4951 & 0.1339 & 0.7175 \\
\hline 6 & 0.01120 & 0.5353 & 0.03222 & 0.7890 & 0.1978 & 0.4965 & 0.1341 & 0.7173 \\
\hline
\end{tabular}

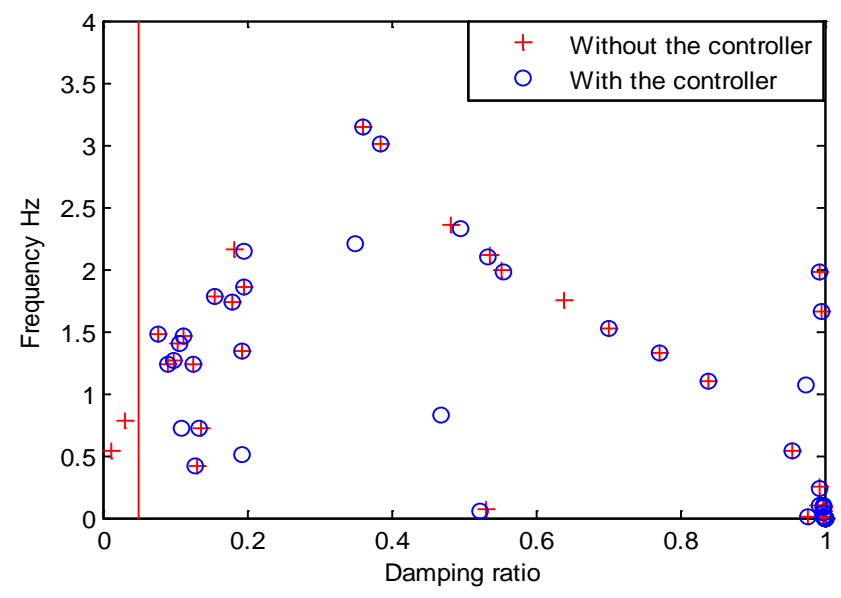

(a)

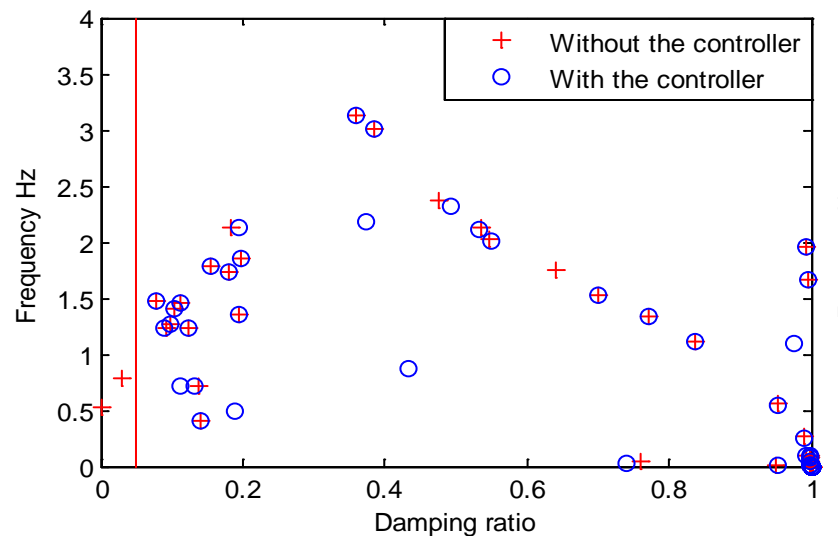

(b)

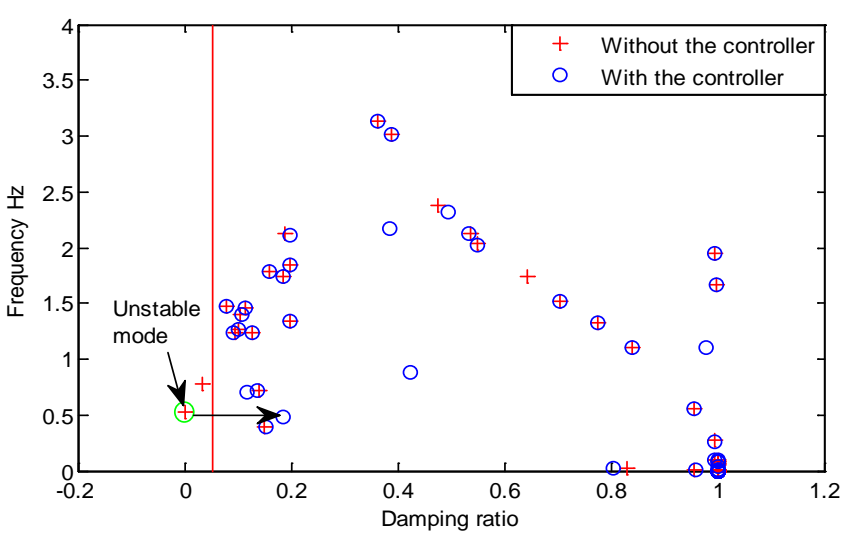

(c)

Figure 11. Modes of the test system under three different operation points. (a) Modes of the test system, OP1; (b) modes of the test system, OP3; (c) modes of the test system, OP4.

controller performance are designed in such a way that different disturbances occur under different operating points and at different parts of the system.

In scenario one, a $50 \mathrm{~ms}$ three phase fault is applied at bus 8 in area 1. Scenario one is applied under operating points 1,3 , and 4 . The resulting tie-line power 
flow through lines 42 through 52 for the operating points 1, 3, and 4 is shown in Figure 12(a)-(c). Rotor angle separation between generators G16 and G1 for scenario one under different operating conditions is shown in Figure 12(d)-(f).

Comparisons between the system with and without the proposed controller show that the maximum overshoot and damping are considerably better with the addition of the proposed controller under all three operating points tested for scenario one. Of particular note are the comparisons for operating point 4 . Without the proposed controller, the system becomes marginally unstable as shown in Figure 12(c) and Figure 12(f). The addition of the proposed controller not only makes the system stable but also damps out oscillations fast. Both the $\mathrm{H}_{2}$ under pole placement and the proposed controller have similar performance; however, in some cases the proposed controller has slightly better damping.

In scenario two, a $50 \mathrm{~ms}$ fault is applied at bus 49 in area 2. This results in significant drop in tie-line flow through lines 42 through 52 during the fault as can be seen in Figure 13(a)-(c). This scenario captures the performance of the proposed controller as the fault is applied relatively close to the SVC. Angular separation between areas 2 and 5 (i.e., between generators G16 and G10) is shown in Figure 13(d)-(f).

\subsubsection{Time Delay}

The major problem with remote signal use is the time delay and the range of the time delay which varies depending on various factors such as the distance of the

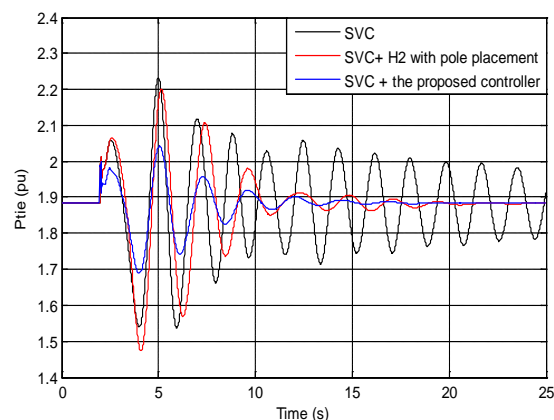

(a)

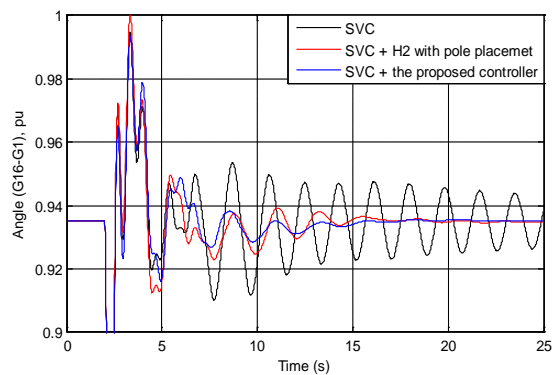

(d)

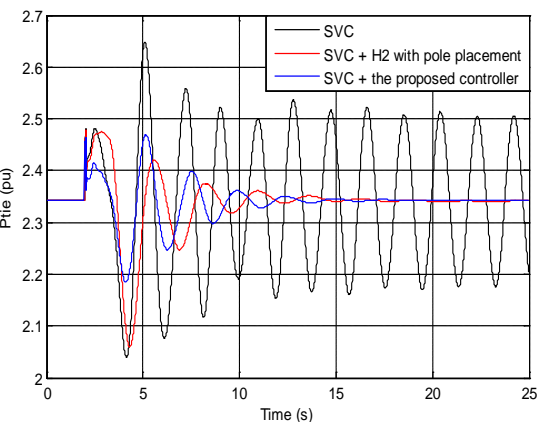

(b)

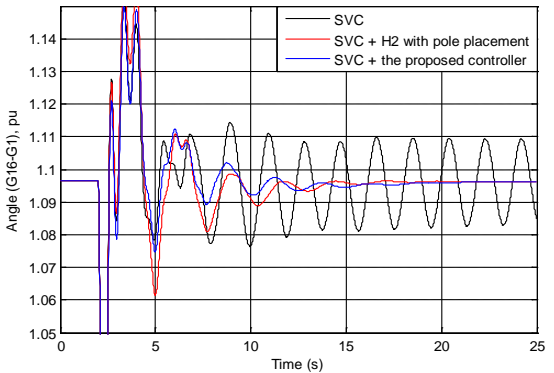

(e)

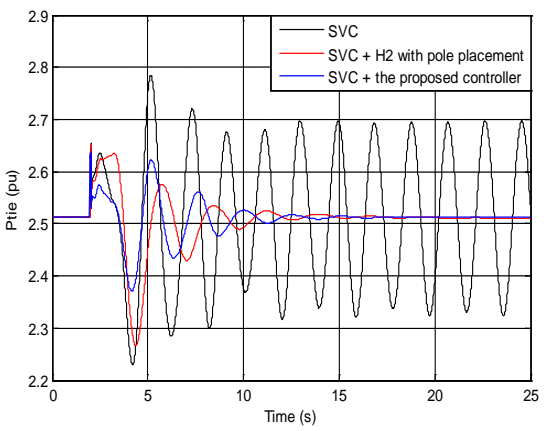

(c)

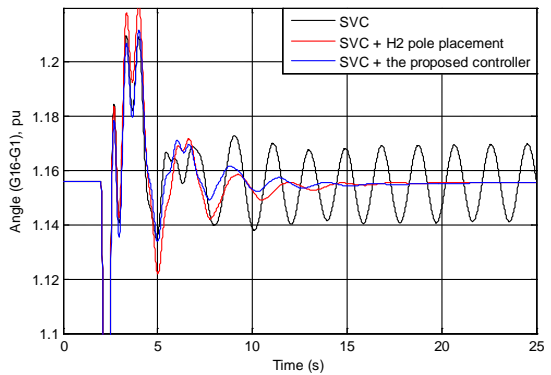

(f)

Figure 12. Dynamic response of the system under three phase fault at bus 8, (Area 1). (a) Tie-line power, OP 1; (b) tie-line power, OP 3; (c) tie-line power, OP 4; (d) angle difference, G16 and G1, OP1; (e) angle difference, G16 and G1, OP3; (f) angle difference, G16 and G1, OP4. 


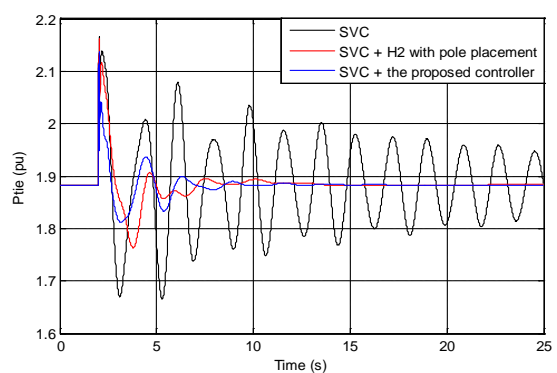

(a)

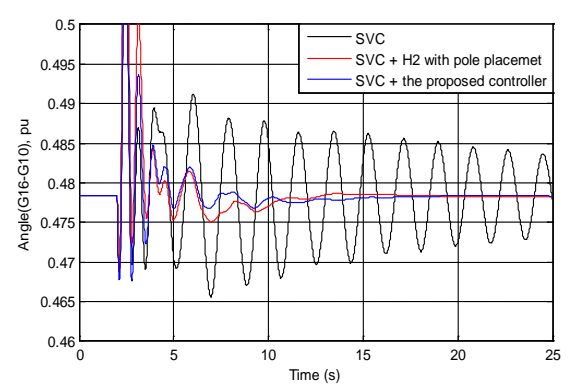

(d)

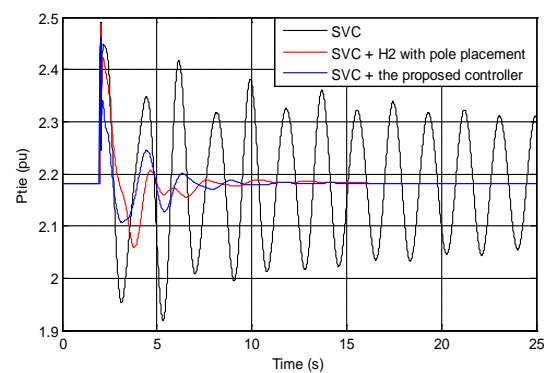

(b)

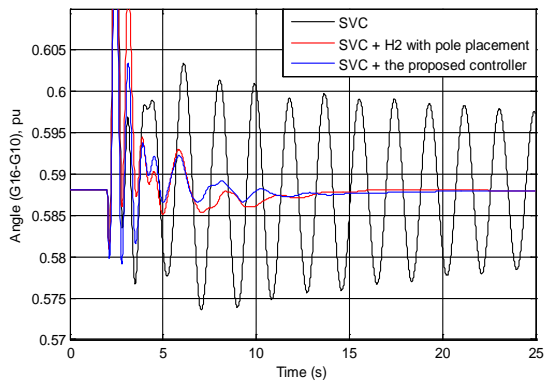

(e)

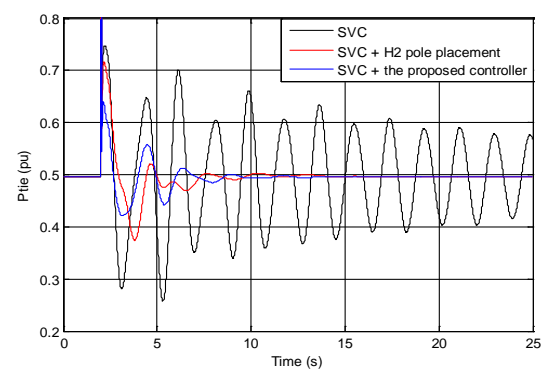

(c)

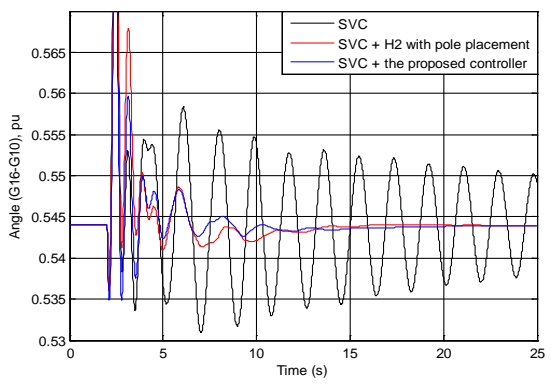

(f)

Figure 13. Dynamic response of the system under three phase fault at bus 49, (Area 2). (a) Tie-line power, OP 1; (b) tie-line power, OP 5; (c) tie-line power, OP 6; (d) angle difference, G16 and G10, OP1; (e) angle difference, G16 and G10, OP5; (f) angle difference, G16 and G10, OP6.

remote signals. These signals can be delayed up to $100 \mathrm{~ms}$. Therefore, it is very important to account for an uncertain time delay to ensure the robustness under various conditions. In this paper, a multi-model optimization method is used to include the effect of time delay. In the previous section, no time delay is considered since the main concern was to show that the method works for different scenarios and is comparable with the existing approach. The time delay in this section has been approximated by the second order Pade approximation [23]. To design a robust controller based on multi-model optimization approach for the uncertainty in time delay, the time delay incorporates the worst case (OP3). Operating points 1 and 2 are also chosen to design the controller. The new controller is designed based on three operating points using the same procedure in section 4 . Figure 14 shows the black diagram for incorporating the time delay with the feedback signal.

Figure 15 shows the dynamic response of the test system with the designed controller for different values of time delay. It can be seen that, the controller is able to damp the power system oscillations under variety of operating points and time delay values. A comparison between the controller designed in section 4 (without incorporating the time delay) and the new controller designed based on incorporating the time delay is shown in Figure 16. Both controllers behave almost the same when the feedback signal is delayed by $200 \mathrm{~ms}$. However, the first controller is not able to damp the inter-area oscillations and the feedback signal is delayed by $300 \mathrm{~ms}$ as shown in Figure 16(b) and Figure 16(d). On the other 


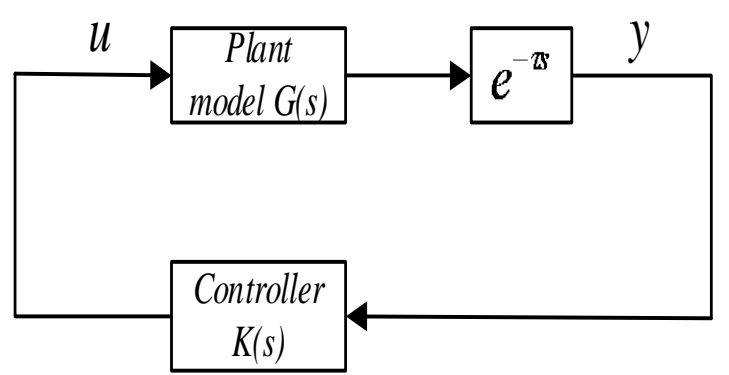

Figure 14. Black diagram of output signal time delay.

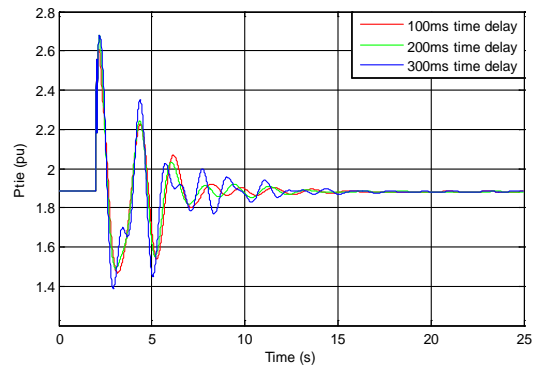

(a)

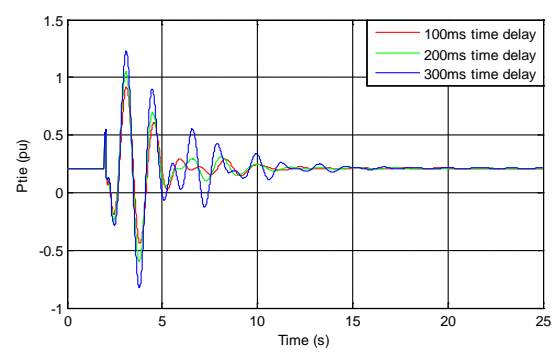

(d)

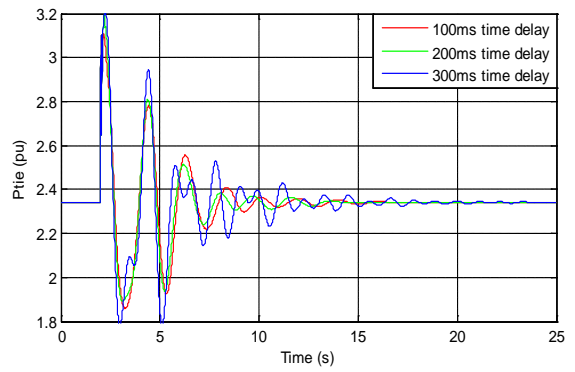

(b)

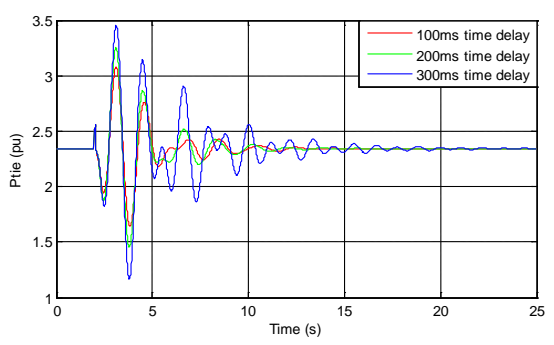

(e)

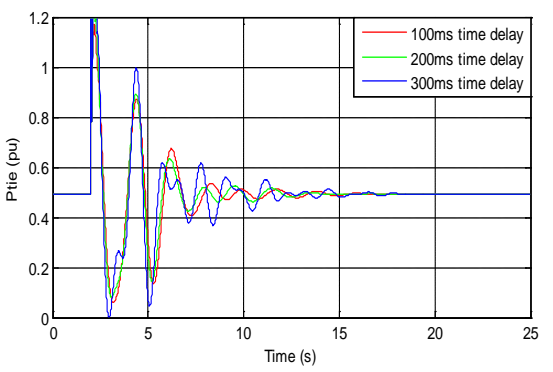

(c)

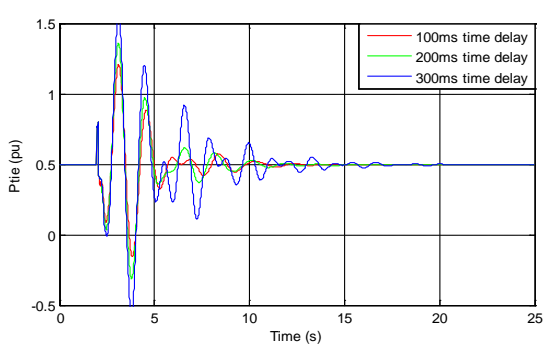

(f)

Figure 15. Dynamic response of the test system with different time delay. (a) Tie-line power, fault at 50, OP 1; (b) tie-line power, fault at 50, OP 3; (c) tie-line power, fault at 50, OP 6; (d) tie-line power, fault at 41, OP 2; (e) tie-line power, fault at 41, OP 3; (f) tie-line power, fault at 41 , OP 4 .

hand, the second controller can maintain system stability and is able to damp these oscillations as seen in Figure 16(b) and Figure 16(d).

\section{Conclusion}

A multi-model approach is used in this paper to design a robust supplementary damping controller. The designed fixed-order supplementary damping controller provides a supplementary signal to the voltage reference set point of SVC. The main objectives achieved in this paper are damping low frequencies oscillations and enhancing power system stability. The controller design relies on shaping the closed-loop sensitivity functions in the Nyquist plot under the constraints of these functions. The IEEE 68-bus system with wind farm is used to demonstrate the controller performance. Test scenarios are designed to emulate real life scenarios seen at system operator level; specifically, uncertainties in operating conditions and changes to system topology are considered. Several test 


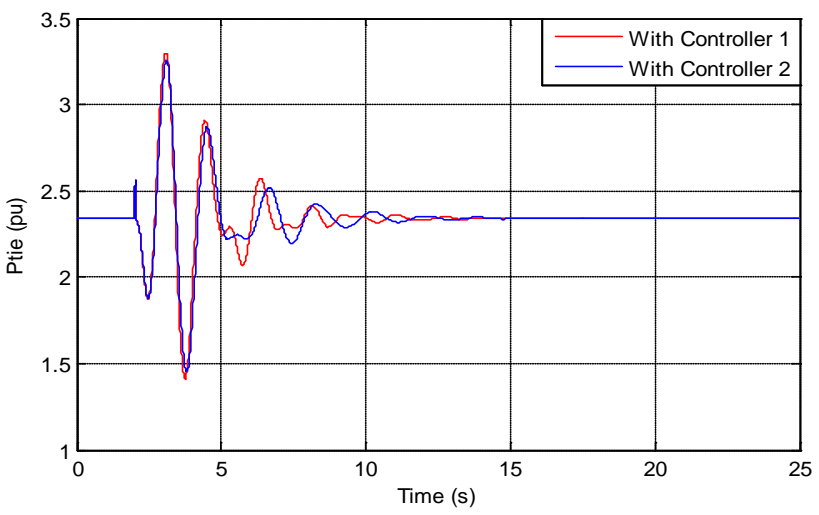

(a)

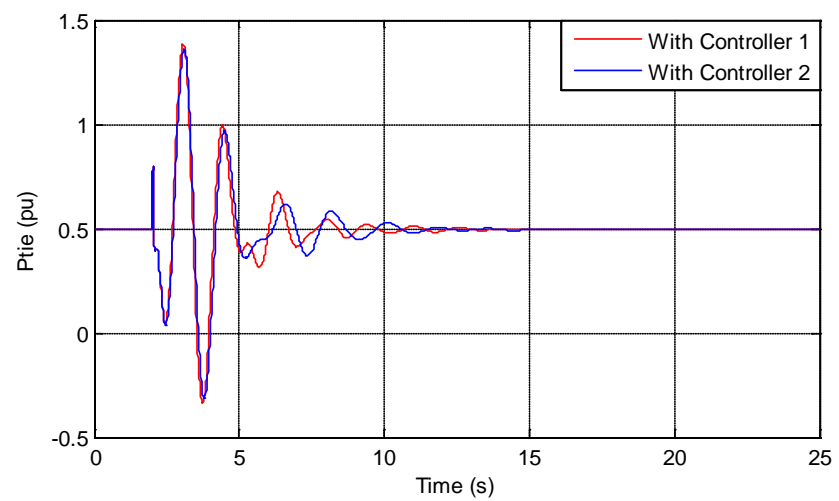

(c)

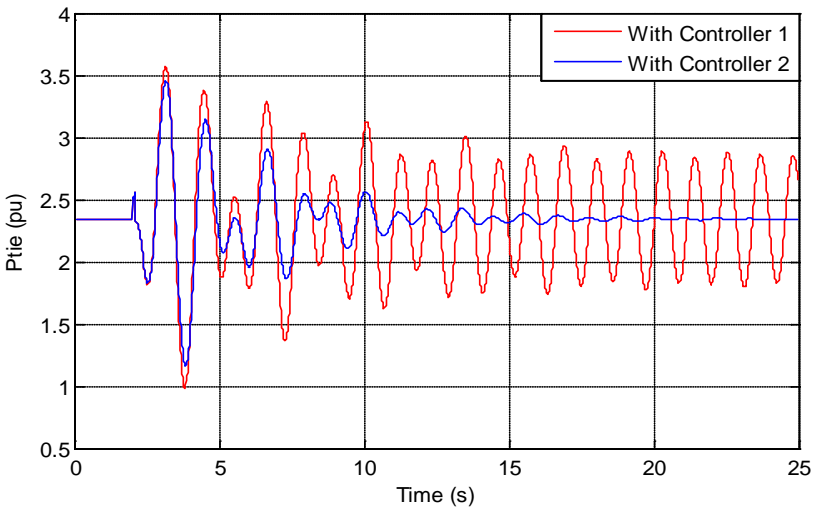

(b)

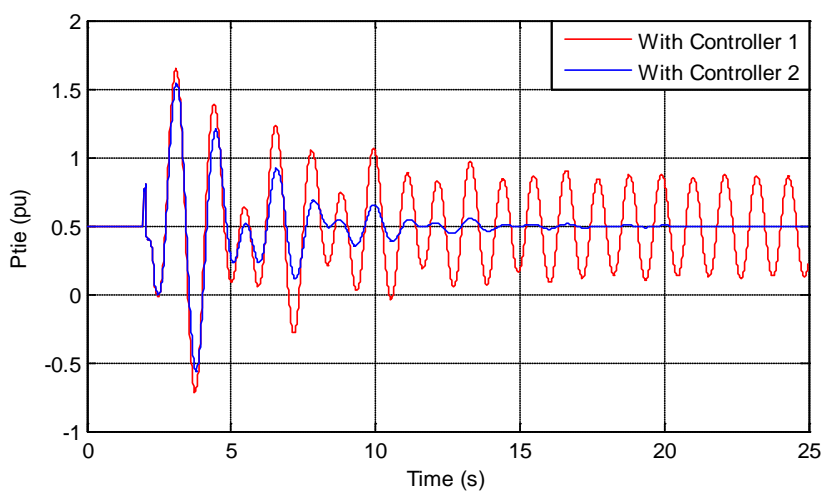

(d)

Figure16. Dynamic response of the test system with the two controllers under different time delay. (a) Tie-line power, fault at 41 , OP $3200 \mathrm{~ms}$; (b) tie-line power, fault at 41, OP $3300 \mathrm{~ms}$; (c) tie-line power, fault at 41, OP $6200 \mathrm{~ms}$; (d) tie-line power, fault at 41, OP $3300 \mathrm{~ms}$.

scenarios are run where disturbances are applied to different areas of the test system under different operating conditions. In all tested cases, the proposed controller significantly improved the system's dynamic response and compared favorably with an existing control technique $\mathrm{H}_{2}$ under pole placement. Improved controller performance with a lower order controller and without the need for model order reduction are the primary advantages of the proposed method that has been validated using both numerical and time domain analysis. The issue of delaying the feedback signal has been addressed using multi-model optimization.

\section{References}

[1] Majumder, R., Pal, B., Dufour, C. and Korba, P. (2006) Design and Real-Time Implementation of Robust FACTS Controller for Damping Inter-Area Oscillation. IEEE Transactions on Power System, 21, 809-816. https://doi.org/10.1109/TPWRS.2006.873020

[2] Zhao, Q. and Jiang, J. (1995) Robust SVC Controller Design for Improving Power System Damping. IEEE Transaction on Power System, 10, 1927-1932. https://doi.org/10.1109/59.476059

[3] Abdlmnam, A., Saraf, P., Balasubramaniam, K., Hadidi, R., Karimi, A. and Makram, 
E. (2016) Design of a Fixed-Order Robust Controller to Damp Inter-Area Oscillations in Power Systems. Journal of Power and Energy Engineering, 4, 61-70. https://doi.org/10.4236/jpee.2016.43006

[4] Chang, Y., Chen, H. and Zhang, F. (2006) Design of Multi-Objective Robust HVDC Supplementary Controller Based on Global Signal. 2006 IEEE PES Power Systems Conference and Exposition, Atlanta, GA, 29 October-1 November 2006, 2146-2151. https://doi.org/10.1109/PSCE.2006.296276

[5] Abdlmnam, A., Saraf, P., Hadidi, R., Karimi, A., Sherwali, H. and Makram, E. (2016) Design of a Fixed-Order Robust Controller Using Loop Shaping Method for Damping Inter-Area Oscillations in Power Systems. 2016 IEEE Power and Energy Conference at Illinois (PECI), Urbana, 19-20 February 2016, 1-6.

[6] Abdlrahem, A.A., Hadidi, R., Karimi, A., Saraf, P. and Makram, E. (2017) Fixed-Order Loop Shaping Robust Controller Design for Parametric Models to Damp Inter-Area Oscillations. International Journal of Electrical Power \& Energy Systems, 88, 164-174. https://doi.org/10.1016/j.ijepes.2016.12.013

[7] Chaudhuri, B. and Pal, B. (2004) Robust Damping of Multiple Swing Modes Employing Global Stabilizing Signals with a TCSC. IEEE Transaction on Power System, 19, 499-506. https://doi.org/10.1109/TPWRS.2003.821463

[8] Klein, M., Le, L., Rogers, G., Farrokpay, S. and Balu, N.J. (1995) Ho Damping Controller Design in Large Power System. IEEE Transactions on Power System, 10, 158-166. https://doi.org/10.1109/59.373938

[9] Chen, S. and Malik, O. (1995) Power System Stabilizer Design Using $\mu$-Synthesis. IEEE Transaction on Energy Conversions, 10, 175-181.

https://doi.org/10.1109/60.372584

[10] Deng, J., Li, C. and Zhang, X. (2015) Coordinated Design of Multiple Robust FACTS Damping Controllers: A BMI-Based Sequential Approach with Multi-Model Systems. IEEE Transaction on Power System, 30, 3150-3159. https://doi.org/10.1109/TPWRS.2015.2392153

[11] Deng, J., Li, C. and Zhang, X. (2014) Robust Damping Control of Power Systems with TCSC: A Multi-Model BMI Approach with $\mathrm{H}_{2}$ Performance. IEEE Transactions on Power System, 29, 1512-1521. https://doi.org/10.1109/TPWRS.2013.2292067

[12] Chaniotis, D. and Pai, M. (2005) Model Reduction in Power Systems Using Krylov Subspace Methods. IEEE Transactions on Power System, 20, 888-894. https://doi.org/10.1109/TPWRS.2005.846109

[13] Sootla, A. (2010) Hankel-Type Model Reduction Based on Frequency Response Matching. IEEE Conference on Decision and Control, Atlanta, 15-17 December 2010, 5372-5377. https://doi.org/10.1109/TPWRS.2005.846109

[14] Pal, B. and Chaudhuri, B. (2006) Robust Control in Power System. Springer Science \& Business Media, Berlin.

[15] Li, Y., Rehtanz, C., Ruberg, S., Luo, L. and Cao, Y. (2012) Wide-Area Robust Coordination Approach of HVDC and FACTS Controllers for Damping Multiple Interarea Oscillations. IEEE Transaction on Power Delivery, 27, 1096-1105. https://doi.org/10.1109/TPWRS.2005.846109

[16] Chaudhuri, B., Pal, B., Zolotas, A., Jaimoukha, I. and Green, T. (2003) Mixed-Sensitivity Approach to H_o Control of Power System Oscillations Employing Multiple FACTS Devices. IEEE Transaction on Power System, 18, 1149-1156. https://doi.org/10.1109/TPWRS.2003.811311 
[17] Alireza, K. and Galdos, G. (2010) Fixed-Order Ho Controller Design for Nonparametric Models by Convex Optimization. Automatica, 46, 1388-1394. https://doi.org/10.1016/j.automatica.2010.05.019

[18] Jonathan, T. (2014) Robust Bode Methods for Feedback Controller Design of Uncertain Systems. Dissertation, Carnegie Mellon University, Pittsburgh, 447.

[19] Chow, J. and Rogers, G. (2008) Power System Toolbox Version 3.0, Ontario.

[20] Milano, F. (2005) An Open Source Power System Analysis Toolbox. IEEE Transactions on Power Systems, 20, 1199-1206. https://doi.org/10.1109/TPWRS.2005.851911

[21] Far, H., Banakar, H., Li, P. and Luo, C. (2009) Damping Interarea Oscillations by Multiple Modal Selectivity Method. IEEE Transactions on Power System, 24, 766-775. https://doi.org/10.1109/TPWRS.2008.2009422

[22] Galdos, G. (2010) Fixed-Order Robust Controller Design by Convex Optimization Using Spectral Models. Thesis EPFL, 4785.

[23] Daniel, D., Silva, A.S. and Decker, I.C. (2009) Wide-Area Measurements-Based Two-Level Control Design Considering Signal Transmission Delay. IEEE Transactions on Power Systems, 24, 208-216. https://doi.org/10.1109/TPWRS.2008.2004733

[24] Karimi, A. (2013) Frequency-Domain Robust Control Toolbox. 52nd Conference on Decision and Control, Florence, 10-13 December 2013, 3744-3749.

https://doi.org/10.1109/CDC.2013.6760460 